\title{
Workshop „Scientist Practitioner - Austausch über Forschungsaktivitäten und wissenschaftliches Selbstverständnis im Fachspezifikum“"
}

\author{
Dienstag, dem 23. Juni 2015 von 11.00 bis 16.30 , Wien
}

\author{
Michael Alexander Wieser · Brigitte Schigl · Henriette Löffler-Stastka \\ Eingegangen: 17. Juli 2015 / Angenommen: 22. Juli 2015 / Online publiziert: 28. August 2015 \\ (C) Springer-Verlag Wien 2015
}

\begin{abstract}
Zusammenfassung Der Beitrag gibt einige Eindrücke und Tendenzen des Workshops wieder, zu dem sich die Veantwortlichen für Wissenschaft und Forschung der Fachspezifika zum Erfahrungsaustausch im Juni 2015 auf Einladung des Bundesministeriums für Gesundheit/Gesundheit Österreich Gmbh in Wien getroffen haben. Das Treffen diente der Vernetzung und Verankerung der Prinzipien wissenschaftlicher Qualität in den Ausbildungseinrichtungen.
\end{abstract}

Schlüisselwörter Wissenschaft und Forschung • Psychotherapie $\cdot$ Fachspezfika Österreich

Diese Arbeit ist Teil des Leitthemas „Psychotherapiewissenschaft“.

M. A. Wieser $(\bowtie)$

Department of Psychology, psychotherapist (psychodrama),

Alpen-Adria-Universitaet Klagenfurt Wien Graz,

Universitaetsstr. 65-67,

9020 Klagenfurt am Woerthersee, Österreich

E-Mail: Michael.Wieser@aau.at

B. Schigl

Rembrandtstraße 4/11,

1020 Wien, Österreich

E-Mail: brigitte.schigl@aon.at

B. Schigl

Schwedengasse 6/A7,

3500 Krems, Österreich

H. Löffler-Stastka

Klinik für Psychoanalyse und Psychotherapie Währinger

Gürtel18-20, Medizinische Universität Wien,

1090 Wien, Österreich

E-Mail: henriette.loeffler-stastka@meduniwien.ac.at
Workshop "Scientist Practitioner: exchange of research activities and scientific self-conception of psychotherapeutic methods" - Tuesday 23 June 2015,11 a. m. to 5.30 p. m., Vienna

Abstract This article reflects some impressions and tendencies of the workshop in June 2015 following an invitation of the Ministry of Health/GÖG in Vienna. The meeting gathered the representatives for science and research together to share their experiences on fostering and implementing science in the psychotherapeutic methods (Fachspezifika). The workshop aimed at networking and anchoring principles of scientific quality in the educational institutions.

Keywords Science and research · Psychotherapy · Psychotherapeutic methods Austria

Um Wissenschaft und Forschung in den psychotherapeutischen Ausbildungsvereinen eine Stimme zu geben und ihren Stellenwert bewusst $\mathrm{zu}$ machen, haben die österreichischen psychotherapeutischen Fachspezifika seit einem Jahr Beauftragte für dieses Thema in ihren Instituten bestimmt. Nun luden die Koordinationsstelle und der Qualitätszirkel Psychotherapieforschung alle diese Beauftragten für Wissenschaft und Forschung zu einem Workshop in die Räumlichkeiten der Gesundheit Österreich GmbH (GÖG) ein.

Maria Sagl eröffnete in Vertretung von Michael Kierein im Namen des Gesundheitsministeriums als Auftraggeber den Workshop und begrüßte die 40 TeilnehmerInnen.

Markus Hochgerner, Leiter des Fachspezifikumsausschusses im Psychotherapiebeirat brachte zur Einstimmung interessante Daten zur Psychotherapie in Österreich. 800 KandidatInnen schließen pro Jahr das Propädeutikum ab, in den fachspezifischen Ausbildungsinstituten sind es 350-400 Menschen, die pro Jahr 
erfolgreich beenden. Das humanistische Cluster hat mit $38 \%$ die meisten KandidatInnen. Psychotherapie ist im österreichischen Gesetz als Heilbehandlung durch Interaktion „mit wissenschaftlich-therapeutischen“ Mitteln grundgelegt. PsychotherapeutInnen sind als Person Handlungswerkzeug - sie führen in bewusst geplanter wissenschaftlich gegründeter Art und Weise ihre Heilbehandlungen durch.

Hochgerner führte weiters aus, wie die Auseinandersetzung mit Anträgen zur Aufnahme als psychotherapeutisches Heilverfahren den Blick vermehrt auf Kriterien wissenschaftlicher Nachweise der psychotherapeutischen Fachspezifika lenkte. Diese Diskussion hat mit dazu beigetragen, dass GÖG den Auftrag bekam, Kriterien der wissenschaftlichen Anerkennung für Psychotherapie zu entwickeln. Der gegenständliche Workshop sei die Gelegenheit, bei dieser Leitlinie zu wissenschaftlichen (Mindest-)Standards und -Empfehlungen partizipativ mit zu gestalten. Diese künftige Leitlinie solle jedes Fachspezifikum für sich (und miteinander) umsetzen mit dem Ziel, theoriegeleitetes, wissenschaftlich fundiertes Handeln auf allen Ebenen zu dokumentieren: In den Erkenntnistheorien, in der Diagnostik, in den Methoden und Techniken der Behandlung, sowie in der Ausbildung ihrer PsychotherapeutInnen.

Gabriele Rieß und Daniela Kern vom GÖG stellten in ihrem Eingangsstatement fest, dass sie seit 2009 als Koordinationsstelle Psychotherapieforschung ein steigendes Bewusstsein für Wissenschaft und Forschung in den Fachspezifika beobachten. Sie erläutern die Ergebnisse einer Vorerhebung zum aktuellen Stellenwert von Wissenschaft und Forschung, an der sich 31 von 41 Fachspezifika beteiligt haben. Wissenschaft und Forschung wird als Qualitätssicherung verstanden aber teilweise auch als Störung der Therapie. Das Spektrum der selbst durchgeführten wissenschaftlichen Arbeiten/Forschung zur Psychotherapie ist breit und reicht von praxisnahen Fallreflexionen bis zu quantitativer Wirksamkeitsforschung. Die Ressourcenfrage spielt dabei eine bedeutende Rolle. Einen wichtigen Schritt stellen die Vorgaben für die Abschlussarbeiten dar. Die Koordinationsstelle Psychotherapieforschung wird eine Datenbank mit laufenden Forschungsprojekten der Fachspezifika einrichten. Die Gestaltung der künftigen Leitlinie zu Wissenschaft und Forschung ist ein wesentlicher Wunsch des Ministeriums.

Daraufhin folgten zwei Impulse des Qualitätszirkels Psychotherapieforschung: Brigitte Schigl und Michael Wieser stellten sich der Frage „Wissenschaft als Handlungsnorm? Reflexionen über ein postmodernes Paradigma und seinen Stellenwert in der Psychotherapie“. Dabei versuchten sie die Wichtigkeit von Wissenschaft bzw. einer bestimmten Art sie zu betreiben zu dekonstruieren. Neben erkenntnis- und wissenschaftstheoretischen Positionen wurden sodann Links zu „big players“ und pragmatische Hinweise und Ressourcen im Bereich der Psychotherapieforschung angeführt.

Nach der Pause berichteten Henriette Löffler-Stastka und Christian Korunka über die im Rahmen des Uni- versitätslehrganges der MedUni Wien entstandenen Forschungsarbeiten, beispielsweise Ausbildungsforschungsstudien $\mathrm{zu}$ Kompetenzen des therapeutischen Handelns im Bereich des humanistischen (Ursula Grillmeier-Rehder) und tiefenpsychologischen Clusters (Hemma Rössler-Schülein). Unter Berücksichtigung internationaler Aktivitäten (z. B. European Association for Psychotherapy) sind Vernetzungsgruppen entlang der Hauptcluster entstanden, die sich mit der Erhebung, Definition, Diskussion von Kernkompetenzen, Operationalisierungsarbeiten zu Veränderungs- und Prozessbeschreibungen und Ethikfragestellungen beschäftigen.

Gabriele Rieß moderierte anschließend ein World Café zum Thema: Stellenwert und Aktivitäten zu Wissenschaft \& Forschung bzw. Implementierung von Wissenschaft \& Forschung im Fachspezifikum. Ziel soll eine erste Annäherung daran sein, welche Aspekte in der Leitlinie des Ministeriums zu Wissenschaft und Forschung fokussiert sein sollen, wie diese für die Fachspezifka auch nützlich sind, und welche Initiativen helfen im Fachspezifikum Wissenschaft und Forschung zu etablieren? Dazu wurden an sechs Tischen mit wechselnden DiskutantInnen in Kleingruppen folgende Fragen bearbeitet.

Im weiteren einige Impressionen aus den Diskussionen zu den einzelnen Fragen:

1. „Was hat sich in den letzten Jahren bzgl. Wissenschaft und Forschung (W\&F) in Ihrem Fachspezifikum (FS) verändert? Welchen Nutzen hatten die bisherigen Unterstützungsangebote?“

Konrad Grossmann berichtete von einem Video Rating nach Schiepek und Kubinger. Zehn Studierende wurden als RaterInnen eingesetzt und dies als Abschlussarbeit oder für je $100 \mathrm{~h}$ im Ausbildungscurriculum anerkannt, die Studienleiter bekamen $600 \mathrm{~h}$ mit halbem LehrtherapeutInnenhonorar vergütet.

Wolfgang Keil erwähnte für die Gesprächstherapie 35 Masterthesen an der Donau-Universität Krems (DUK) und der Implementierung von zwei Forschungsseminaren im Curriculum.

Einige KollegInnen erzählen von schon gebahnten Kooperationen für Forschungsaktivitäten:

Otmar Wiesmeyer (Existenzanalyse und Logotherapie) arbeitet mit der DUK als auch der Universität Osnabrück in Sachen Forschung zusammen.

Ingeborg Netzer von der Gestaltsektion des Österreichischen Arbeitskreises für Gruppentherapie und Gruppendynamik (ÖAGG) kooperiert ebenfalls mit der DUK bei Forschungsprojekten.

Machowetz-Müllner teilte mit, dass Neuro-Linguistisches-Programmieren (NLP) eine Metastudie durchführt.

Nach Nestor Kapusta arbeitet die Individualpsychologie mit MedUni Wien, Uni Wien und der Selbstpsychologie für wissenschaftliche Projekte zusammen.

2. „Welchen aktuellen Stellenwert hat W\&F im FS (Kriterien/Struktur, Aktivitäten/Kooperationen, For- 
schungsthemen)? Welche Möglichkeiten und konkreten (Umsetzungs-)Pläne haben Sie im FS? Welche Herausforderungen sind zu bewältigen?"

Susanne Ohmann und Evelyn Schmied-Wadda bereiten einen Kongress für Verhaltenstherapie vor. Für Forschung gibt es derzeit keine Bezahlung, jedoch wurde die Ausbildungsordnung geändert, um Forschungsvorhaben zu unterstützen bzw. zu ermöglichen.

In der Gestalttherapie (Ursula Grillmeier Rehder) sind Forschungen zum Thema Beziehung und Grundhaltung im Vordergrund, die spezifisch gestalttherapeutischen Kompetenzen werden ausgearbeitet.

Klaus Schulte (dynamische Gruppentherapie) baut im ÖAGG eine Forschungsambulanz auf.

Brigitte Macke-Bruck (personenzentrierte Psychotherapie) arbeitet an spezifischen Fragen zur therapeutischen Methode und berichtet von clusterspezifischen Vernetzungstreffen.

Silke Gahleiter (Integrative Therapie, DUK) will eine Ausgewogenheit von Forschungsinteresse und Mittel erreichen.

Henriette Löffler-Stastka betonte die Notwendigkeit eines konkreten Arbeitsprofils und von Zieldefinitionen für die Weiterentwicklung der Forschungsbeauftragten.

3. „Welche Visionen bzgl. W\&F (Aktivitäten, Kooperationen) haben sie und welche (externen) Maßnahmen zur Implementierung von $W \& F$ wären hilfreich (Strukturvorgaben, Ausbildungs-Curriculum, etc.)? Woran erkennen Sie (könnten Sie in Zukunft ... erkennen) im FS die Implementierung von W\&F? Was empfehlen Sie Ihrem FS für die Implementierung von W\&F?"

Elfriede Wieser von der Transaktionsanalyse (DUK) brachte das Thema Anonymisierung bei Forschung ein.
Karin Brem-Gintenstorfer plädierte dafür die Didaktik in der Ausbildung nicht aus den Augen zu verlieren.

Sodann trafen sich die von den Tischgruppen gewählten ModeratorInnen zum Plenumsbericht: Dabei wurden Wünsche nach mehr qualitativer und soziologischer Forschung, einem Psychotherapieforschungsfonds und einem Literatur-Datenbankzugang formuliert. Die Anforderungen an die Lehrenden wurden ebenso genannt, wie eine vermehrte Einbindung der PraktikerInnen in die Forschungsbemühungen. Gemeinsame Publikationen könnten dabei helfen. An einigen Tischen wurde eine Fachspezifika-übergreifende, interdisziplinäre Forschungskultur gewünscht, in der gemeinsame Projekte realisiert werden könnten. Vernetzungsprojekte innerhalb der Cluster, mit Universitäten und international wurden angedacht. Dazu sei Beratung und Unterstützung der Koordinationsstelle - wie etwa Zugang zu Datenbanken - gewünscht. GastdozentInnen für wissenschaftliche Forschung könnten von mehreren Ausbildungsinstituten gemeinsam engagiert werden. Betont wurde allseits der Wunsch, die österreichische Vielgestaltigkeit in der Psychotherapielandschaft zu erhalten - dazu sei ein breiter Wissenschaftsbegriff, der alle vier Cluster umschließt, nötig. Realistisch sei mehr praxisorientierte Forschung als Grundlagenforschung.

Die Partizipationsmöglichkeit zur Erstellung einer Leitlinie Psychotherapieforschung wurde von Markus Hochgerner abschließend noch einmal betont, ca. 2017/2018 soll es eine Umsetzung dieser Leitlinie in allen Fachspezifika geben.

Es bleibt zu hoffen, dass die Fachspezifika diesen Auftrag annehmen und sich weiter um die Implementierung von Wissenschaftlichkeit in ihren Verfahren und deren Ausbildungen bemühen. Die vom Bundesministerium für Gesundheit beauftragte GÖG/Koordinationsstelle mit dem Qualitätszirkel Psychotherapieforschung wird das ihre dazu tun, die Fachspezifika darin zu unterstützen. 